\title{
Diabetes mellitus: Plasticizers and nanomaterials acting as endocrine-disrupting chemicals (Review)
}

\author{
MIHAELA JANA TUCULINA ${ }^{1 *}$, PAULA PERLEA ${ }^{2}$, MIRCEA GHEORGHIȚA $^{1}$, CRISTIAN NIKY CUMPĂTĂ $^{3 *}$, \\ IONELA TEODORA DASCĂLU ${ }^{1}$, ADINA TURCU ${ }^{1}$, ANDREEA GABRIELA NICOLA ${ }^{1 *}$, \\ LELIA MIHAELA GHEORGHIȚA ${ }^{1}$, OANA ANDREEA DIACONU ${ }^{1 *}$, ANA VALEA ${ }^{4}$, \\ ADINA GHEMIGIAN $^{5,6}$ and MARA CARSOTE ${ }^{5,6}$
}
${ }^{1}$ Department of Orthodontics, Faculty of Dental Medicine, University of Medicine and Pharmacy of Craiova, 200349 Craiova;
${ }^{2}$ Department of Endodontology, Faculty of Dental Medicine, 'Carol Davila' University of Medicine and Pharmacy, 050474 Bucharest; ${ }^{3}$ Faculty of Dental Medicine, 'Titu Maiorescu' University of Bucharest, 031593 Bucharest; ${ }^{4}$ Departement of Endocrinology, 'Iuliu Hatieganu' University of Medicine and Pharmacy, 400337 Cluj-Napoca;
5 Department of Endocrinology, 'Carol Davila' University of Medicine and Pharmacy, 020021 Bucharest;
${ }^{6}$ Department of Endocrinology, 'C.I. Parhon' National Institute of Endocrinology, 011863 Bucharest, Romania

Received October 19, 2021; Accepted November 18, 2021

DOI: $10.3892 /$ etm.2022.11217

\begin{abstract}
Various plasticizers and nanomaterials have been linked to endocrine disruptors or endocrine-disrupting chemicals (EDCs) which represent a large, heterogeneous, yet incompletely understood group of structures acting on normal and pathological body pathways such as hormonal production, secretion, transport and receptor binding. By contrast, various
\end{abstract}

Correspondence to: Professor Paula Perlea, Department of Endodontology, Faculty of Dental Medicine, 'Carol Davila' University of Medicine and Pharmacy, 17-23 Calea Plevnei Street, 050474 Bucharest, Romania

E-mail: paula.perlea@gmail.com

Professor Ionela Teodora Dascălu, Department of Orthodontics, Faculty of Dental Medicine, University of Medicine and Pharmacy of Craiova, 2-4 Petru Rareș Street, 200349 Craiova, Romania

E-mail: marceldascalu@yahoo.com

*Contributed equally

Abbreviations: BPA, bisphenol A; COVID-19, Corona Virus Disease-19; CADD, computer-assisted drug design; CHAMACOS, Center for the Health Assessment of Mothers and Children of Salinas; DM, diabetes mellitus; EDC, endocrine disrupting chemicals; ER, endoplasmic reticulum; FSH, follicle stimulating hormone; FoxM1, Forkhead box protein M1; GLP-1, glucagon-like peptides; IRS1, insulin receptor substrate 1; MAP, modified apple polysaccharide; MSN, mesoporous silica nanoparticle; Pd-NPs, palladium nanoparticles; pSTAT1, phosphorylated signal transducer and activator of transcription 1; RACK1, receptor for activated C kinase 1; ROS, reactive oxygen species; $\mathrm{SiO} 2 \mathrm{NPs}$, silicon dioxide nanoparticles; SeNPs, selenium nanoparticles; VLP, virus-like particles

Key words: plasticizers, nanomaterial, endocrine disruptors, diabetes mellitus, phthalates, bisphenol A applications of nanoparticles are currently under investigation since the delivery of useful drugs, particularly insulin in diabetes mellitus, is essential in case of insulin deficiency. The aim of the present review was to introduce and examine different plasticizers and nanomaterials with potential applications for diabetic patients (such as selenium or gold-based nanoparticles that help the oral delivery of insulin) or plasticizers/nanomaterials acting similarly to EDCs in relation to the human and animal body, particularly glucose metabolism impairment such as diabetes mellitus (DM). Bisphenol A is a chemical used worldwide; however, the effect of exposure varies with regard to the source, environment, time of exposure and the age of the organism. Daily exposure is mostly related to food and drinks stored in polycarbonate plastics. However, exposure may also be through the skin or through the maternal placenta or breast milk which are risk factors for the fetus and for the newborn. It exerts an estrogen-like profile, but it also induces insulin resistance by impairing peripheral insulin receptors or it decreases insulin secretion by acting at the level of insulin-secreting pancreatic $\beta$-cells. Phthalates, compounds of flexible plastics, act as EDCs via their human metabolites such as diethyl phthalate and derivative monoethyl phthalate. Their role in inducing gestational DM and weight gain/obesity during pregnancy has been showcased. The vast field of plasticizers and nanomolecules acting as endocrine disruptors is widely linked to clinical aspects of DM, a serious condition with a major population impact. The importance of understanding and using these agents and applications is reflected in saving numerous human lives.

\section{Contents}

1. Introduction

2. Diabetes mellitus (DM): Past and future

3. Conclusions 


\section{Introduction}

Various nanomaterials have been linked to endocrine disruptors or endocrine-disrupting chemicals (EDCs) which represent a large, heterogeneous, yet incompletely understood group of structures acting on normal and pathological body pathways such as hormonal production, secretion, transport, activation/inactivation, receptor binding and feedback regulation (1-3). This is an ongoing topic of discussion due to a high variety of nanotechnology-related molecules that mimic, block or antagonize the organs or tissues of humans and/or animals and thus an increase or a deficiency of physiological organism pathways of regulation is identified (1-3). EDCs are part of pollutants that dysregulate the functions of cells representing an emerging public health issue (1-3). However, a large amount of data remain a matter of debate or remain incompletely known while a close collaboration of biotechnology specialists with clinicians and researchers of different medical fields is essential on this particular topic (1-4). Other areas that actually have a lack of feasible information are the methods and protocols existing thus far to routinely assess the effect of EDCs on human and animal bodies (5). It only takes a small amount of a certain EDC to alter an entire signal transduction network, and methods used vary from traditional well-known instruments such as enzyme-linked immunosorbent assay (ELISA) or high-performance liquid chromatography (HPLC) to modern electrochemical detectors based on biosensors using different nanomaterials (5).

An extensive number of EDC-associated disorders vary from digestive and endocrine cancers, inflammatory conditions to autoimmune diseases, and DM but mostly the mechanisms coexist at multiple levels. For instance, EDC-emerged inflammatory and destructive antibodies in the pathogenesis of neoplasia, infertility as well as DM are to mention but a few (1-3), (Fig. 1). In fact, EDCs regulate the microenvironment of both estrogenic and androgenic receptors and the immune system activation through signal proteins such as receptor for activated C kinase 1 (RACK1), a promoter of carcinogenesis (1). Another largely described family of EDCs acting as carcinogenic promoters is represented by bisphenols which are identified in various products; they impair multiple biological functions such as hormonal secretion or cell growth, including steroid receptors (2). By contrast, enzymatic nanoreactors such as virus-like particles (VLP) identify the EDC-induced damage at the level of natural defense chains including activity of cytochrome P450 enzymes involved in oxidative stress and glucose metabolism (3). Exposure to industry-derived palladium nanoparticles (Pd-NPs) reveals EDC potential, particularly by impairing the gonadal axes (3). Wistar rats exposed to Pd-NPs for a prolonged period of time in small amounts had higher levels of follicle stimulating hormone (FSH), as negative feedback to ovarian failure which decreases the reproductive potential (4). In general, a need for interventional strategies of biophysics detection and medical risk evaluation of nanomolecular-based EDCs is required knowing that actually most advanced assessment methods are also based on nanotechnology (1-5).

By contrast, various applications of nanoparticles are currently under investigation, since the delivery of useful drugs, particularly insulin for DM is essential for individuals with insulin deficiency.

\section{DM: Past and future}

The present review aimed to introduce and assess different plasticizers and nanomaterials with potential applications in the everyday life of diabetic patients or structures acting similarly to EDCs in association to human and animal organisms, particularly at the level of glucose metabolism impairment such as in DM. This a narrative review; 50 studies are cited, published between 2014 and 2021. Full length English articles were included. A comprehensive PubMed search was conducted with the following search terms: 'Endocrine disrupting chemicals', 'diabetes mellitus', 'nanomaterials', and 'plasticizers' in different combinations. The selection of cited studies was based on the most relevant articles which highlight key transdisciplinary information, combing through both clinical points and chemical/biophysics features. Each key point is identified and presented in the following subsections.

DM: A global concern. Glucose metabolism anomalies vary from impaired glucose tolerance, impaired fasting glucose to frank DM of different types such as type 1, type 2, and secondary type, associated to endocrine conditions such as acromegaly or Cushing syndrome (6). A significant number of complications are associated with the disease including cardiovascular risk, neurological conditions (such as stroke and neuropathy), kidney failure, obesity, high blood pressure, polycystic ovary syndrome, infertility, hypogonadism, sarcopenia, higher risk of infections, certain malignancies, depression, hypovitaminosis $\mathrm{D}$, osteoporosis in addition to overall reduced quality of life and increased mortality (6,7). Diabetes is closely associated with obesity contributing to the concept of 'diabesity' which, at least in pre-pandemic days, was considered the true global pandemic of the world $(8,9)$. Diabetes is considered the true pandemic of the modern era, at least before the Corona Virus Disease-19 (COVID-19) pandemic wave that has affected the population worldwide $(8,9)$.

The increasing incidence of DM over the last decades, still highly underestimated in the general population, has generated increased economic and social burden, thus rendering it important to study the potential molecules involved in its pathogeny and new efficient treatment options $(8,9)$. A total of $1 / 11$ adults is diagnosed with DM (90\% have type 2 DM) $(6,8,9)$. Globally, in 2000 there were an estimated 151 million diabetic individuals, while the prediction for 2030 was 324-366 million individuals, depending on the source, but this was actually demonstrated to be an underestimation since in 2015 there were already 415 million individuals diagnosed with the condition $(6,8,9)$.

Multiple factors play a certain role in the pathophysiological mechanisms of DM including lifestyle choices (sedentary habits and junk food consumption), genetic susceptibility (such as genes involved in glucose metabolism, insulin control and secretion), chronic inflammation, oxidative stress, growth hormone and cortisol axis anomalies which contribute to insulin resistance and are a necessary step in type 2 DM development $(6,8,9)$. EDCs have been demonstrated to be involved in the occurrence of DM based on experimental and clinical studies (8-10). For instance, bisphenol A (BPA) induces excessive insulin secretion impairing the communication among fat-muscle-liver-pancreas (10). Other molecules may display a 


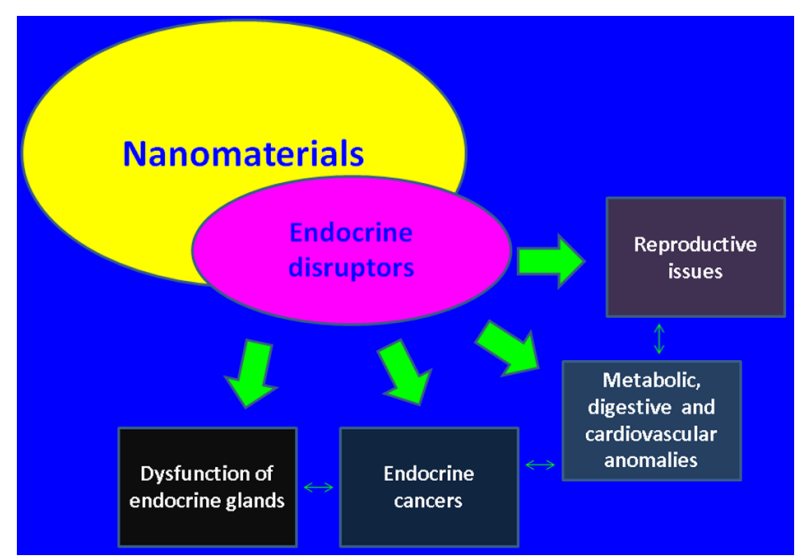

Figure 1. Association of nanomaterials, as endocrine disruptors, with endocrine and metabolic issues in human and animal organisms.

similar effect including pesticides, dioxins, and aromatic polycyclic hydrocarbides (10). Certain EDCs are nanomolecules with varying applications from industry or medical fields (10).

Diabetes and nanotechnology. Nanotechnology has been linked to diabetes, either as unwelcome side effects of nanomolecules that cause glucose profile damage, or as applications, for instance, of insulin delivery devices into the body or nanoparticles acting as hydrogels that promote wound healing based on attached bioactive molecules such as growth factors or proteins (11). Transdisciplinary nanotechnology involving diabetes is expansively developing due to the major epidemiological effect in the general population and it can be applied through the use of traditional protocols of diagnosis and therapy and also by using advanced computer-assisted drug design (CADD) chemoinformatics instruments (12). However, numerous questions remain unanswered on this topic, including the exact relationship between endocrine disruptors and their effects on humans and animals, the exposure time required in order to obtain a particular effect and the interassociations between genetic background and environmental exposure. In addition, the level of evidence is low in multiple areas, particularly in type $2 \mathrm{DM}$ at various ages, in relationship to a certain anti-diabetic regime of medications, etc. Potential mechanisms of nanomaterial-based EDCs which damage glucose metabolism are introduced in Fig. 2.

Selenium nanoparticles (SeNPs) and insulin delivery. Among inorganic nanoparticles, selenium incorporation represents an extension of their well-known standards regarding reduced toxicity, increased bioactivity and improved targeting (13). SeNPs may improve the negative aspects of selenium delivery which include a limited and narrowed therapeutic window and easily achievable toxicity levels (13). However, this essential trace element is very useful in the human organism since it plays a major role in the active core of multiple enzymes called selenoproteins which are mostly associated with oxido-reductase functions (13). Thus, SeNPs are currently being explored in applications for conditions related to chronic exposure of oxidative stress as observed in chronic inflammation such as cancer or diabetes (13). In addition, SeNPs may

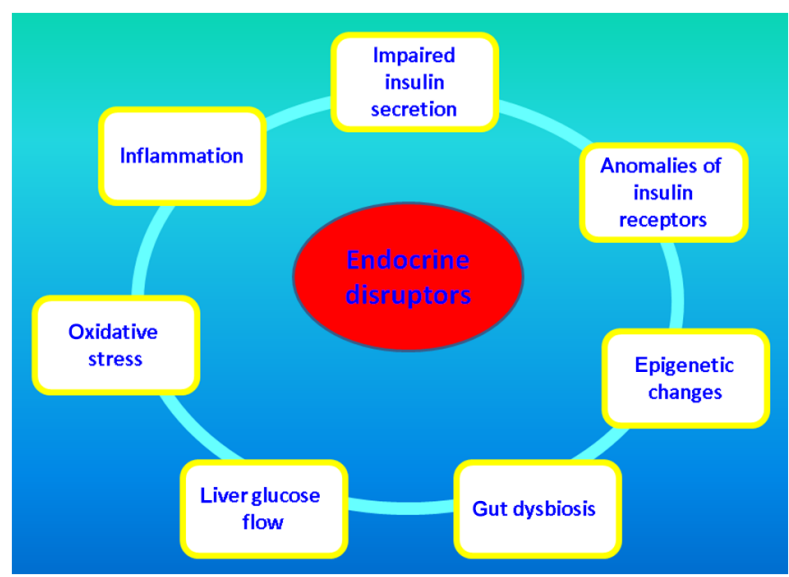

Figure 2. Potential mechanisms of endocrine disruptors involved in impaired glucose metabolism, particularly in type 2 diabetes mellitus.

serve as pharmacological carriers in various medical conditions even if the exact kinetics of such mechanisms remain insufficiently known (13). An example of this, is the delivery of oral insulin in type 1 (insulin-dependent DM) or type 2 DM $(13,14)$. Oral insulin has an extremely poor bioavailability and insulin-loaded SeNPs may overcome the absorption limits releasing a controllable amount of the hormone ( $9 \%$ bioavailability of subcutaneous insulin in murine experiments) with high stability in the digestive environment (15).

Gold nanoparticles and insulin delivery. Nanoparticles containing gold have the advantage of reduced toxicity and increased surface area which render them useful in drug delivery such as in insulin for glucose control or growth factors for diabetic wound healing using various nanotechnology-based devices (16). This type of nanoparticle is listed, as well as SeNPs, among future insulin nanocarriers, a large family that also includes liposomes, dendrimers, niosomes, micelles, which are currently under evaluation indicating a promising and remarkable option in the management of DM (17). For instance, a study from 2020 used gold nanoparticles based on acid chloroauric reduction by modified apple polysaccharide (MAP) in association with insulin, for oral delivery in rats with streptozotocin-induced DM (18). Nanotechnology-based insulin caused a 3.36-fold decrease in glycemic levels within 240 min compared with oral non-conjugated insulin, an effect that was consistent after 28 days in improvement of other glucose-related parameters such as blood lipids and the weight of rats (18).

Glucose-responsive insulin delivery devices. Systems which are able to achieve a constant level of glycemia by regulating insulin release appear to be more promising options than those that exist to date, as far as DM therapy is concerned (19). Such a device is mesoporous silica nanoparticle (MSN)-based with self-regulation (19). Insulin is attached to the channels of MSNs via adsorption and it is released depending on the needs of diabetic mice during a 12-h interval (19). Integrating sensors of real-time blood glucose (such as Alizarin complexone) to an MSM-insulin system helps the adequate release of the hypoglycemic hormone (20). 
Glucagon-like peptide (GLP) analogues and nanotechnology. GLP analogues (such as GLP-1) represent a new generation of hypoglycemic agents with real benefits not only to glucose control, but also to reduction of diabetes-associated cardiovascular risk (21). This class of drugs has a similar issue as human insulin, which is a low bioavailability if the oral route is used, thus the need for new means of delivery (21). Nanotechnology has taken an important step in resolving this matter by introducing carrier nanoparticles that increase bioavailability of GLP-1 analogues (22). For instance, cyclodextrin and liraglutide (a GLP-1 analogue) containing nanocarriers exhibited a high stability in the intestinal site offering a protection of GLP-1 analogue from enzyme degradation up to $4 \mathrm{~h}$ in murine experiments (22).

Diabetes wound healing and nanotechnology. DM is associated with a very high risk of wounds, skin infections and foot ulcers, with poor healing due to a multifactorial etiology (11). Nanotechnology devices that deliver wound healing drugs are based on nanoparticles/hydrogels in addition to bioactive pharmacological products (11). For instance, a recent in vivo study which incorporated silver ions to a chitosan hydrogel to deliver epidermal growth factor with antibacterial and cell growth effects in diabetic mice, translated into encouraging clinical effects such as re-epithelization of the area of the ulcer and increased collagen deposits in the same foot region (23). An alternative for chronic skin diabetic lesions is a system containing hyaluronic acid in combination with oxidized hydroxymethyl propyl cellulose and oridonin-loaded alginate microspheres, which promotes fibroblastogenesis and angiogenesis and reduces inflammation by inhibiting factors such as interleukin-6 (24).

Silicon dioxide nanoparticles (SiO2NPS) and insulin resistance. $\mathrm{SiO} 2 \mathrm{NPs}$, food industry-related EDCs, are studied in murine experiments to induce insulin resistance (25). Oral intake of SiO2NPs (on doses calculated based on the body weight of mice) induces hyperglycemia after they are absorbed and act through glucose transporters and insulin receptors at the level of organs involved in insulin activity such as the liver, or insulin secretion such as the pancreas (25). The underlying mechanisms include upregulation of genes encoding reactive oxygen species (ROS) production via increasing the level of stress at the endoplasmic reticulum (ER) (25). ROS activate the NF-kB pathway leading to increased cytokines that promote inflammation further inducing insulin resistance due to serine phosphorylation of insulin receptor substrate 1 (IRS1) (25).

Gut microbiome as a target of EDCs and promoter of metabolic anomalies. Previous studies have indicated that gut dysbiosis is an important source of metabolic anomalies including DM, obesity, immune and autoimmune conditions as well as cancer $(25,26)$. Due to the multitude of microorganisms that inhabit the gut microbiome, this environment is extremely dynamic and heterogeneous and it is targeted by molecules originating from outside of the body, including EDCs, which stimulate the negative effects associated to microorganism proliferation and virulence, including the production of lipopolysaccharides or the induction of epigenetic changes in host human organisms (26).
$B P A$. BPA is a well-known EDC and certain studies have revealed that even at nanomolecular doses it induces negative effects on human and animal organisms (27,28). BPA is a chemical used worldwide; however, the exposure varies with regard to the source, environment, time of exposure and age of the organism $(27,28)$. Daily exposure is mostly associated to food and drink containers sold on the market, as well as absorption through the skin or through the maternal placenta or breast milk which are risk factors for the fetus and for the newborn baby $(27,29)$. It exerts an estrogen-like profile but it also induces insulin resistance by impairing peripheral insulin receptors or it decreases insulin secretion by acting on insulin-secreting pancreatic $\beta$-cells $(27,30)$. Insulin resistance as well as impaired pancreatic insulin secretion are key pathogenic elements in type 2 DM $(27,31)$. BPA may also be a missing element in the complex pathogenesis of obesity, also with a major epidemiological impact in modern society, which currently has yet to be completely elucidated $(27,32)$.

The chemical xenoestrogen, a major component of multiple plastic products of various domestic and industrial sources, aggravates glucose control in addition to fatty liver disease and impairment of hypothalamic pathways involved in the regulation of energy balance through food intake $(33,34)$. Other EDCs with central effects are phthalates, biphenyls and tributyltin $(35,36)$. The effect of epoxy resins and polycarbonate plasticizers containing BPA may be quantified by assessment of free BPA levels in urine samples based on different methods such as solid-phase extraction $(37,38)$. Higher levels in diabetic patients are an indicator of their role in the pathogeny of diabetes $(39,40)$. However, according to current knowledge, DM, as well as obesity are regarded as pluri-factorial and systemic conditions, and the role of BPA needs to be integrated in a more complex frame of genetic, epigenetic and environmental elements $(41,42)$.

Prenatal exposure to phthalates. Phthalates, compounds of flexible plastics, act as EDCs via their human metabolites such as diethyl phthalate and derivative monoethyl phthalate $(43,44)$. It has been indicated that they participate in inducing type 2 DM as well as gestational DM and weight gain/obesity during pregnancy $(45,46)$. However, the exact pathogenic mechanisms remain a matter of debate; for instance, the Center for the Health Assessment of Mothers and Children of Salinas (CHAMACOS) cohort study published in 2021 on 415 pregnant women, based on the assessment of 11 urinary metabolites (using mass spectrometry), revealed that the direct association with the glucose profile was not statistically significant, however it was with weight gain which is an indirect contributor to insulin resistance as a potential contributor to DM (47). Murine experiments revealed another mechanism involving insulin-secreting pancreatic $\beta$-cells: Di-n-butyl phthalate upregulated the expression of phosphorylated signal transducer and activator of transcription 1 (pSTAT1) which inhibited Forkhead box protein M1 (FoxM1), as a toxicity mediator of $\beta$-cell dysfunction (48). Additional effects of plastic bottles and plastic containers of foods and drinks are transferred to the fetus producing disturbances in embryonic development and growth restriction, due to the epigenetic role of phthalates ingested by pregnant women (49). In addition, 
the phthalate-related risk is identified later in life; for example, pubertal mice with type 2 DM displayed neurotoxicity when exposed to oral ingestion of di-2-ethylhexyl phthalate for three weeks; the presence of glucose anomalies being prone to further neuronal damage (50).

\section{Conclusions}

The vast field of plasticizers and nanomolecules acting as endocrine disruptors is widely linked to the clinical aspects of DM, a serious condition with a major impact worldwide. The importance of understanding and using these agents and applications is reflected in saving numerous human lives.

\section{Acknowledgements}

Not applicable.

\section{Funding}

No funding was received.

\section{Availability of data and materials}

Not applicable.

\section{Authors' contributions}

PP and ITD revised the manuscript and are the corresponding authors. MC summarized the literature findings and wrote the manuscript. MJT, MG, CNC, AT, AGN, LMG and OAD revised the literature data. $\mathrm{MG}, \mathrm{CNC}, \mathrm{AT}, \mathrm{AGN}, \mathrm{LMG}, \mathrm{OAD}$, $\mathrm{AV}$ and $\mathrm{AG}$ researched the articles that were included as references. MJT, PP, ITD, MG, CNC, AT, AGN, LMG and OAD reviewed the literature findings, critically revised the manuscript and approved the current form of the review. All authors read and approved the published version of the manuscript. Data authentication is not applicable.

\section{Ethics approval and consent to participate}

Not applicable.

\section{Patient consent for publication}

Not applicable.

\section{Competing interests}

The authors declare that they have no competing interests.

\section{References}

1. Buoso E, Masi M, Racchi M and Corsini E: Endocrine-Disrupting Chemicals' (EDCs) effects on tumour microenvironment and cancer progression: Emerging contribution of RACK1. Int J Mol Sci 21: 9229, 2020.

2. González-Davis O, Chauhan K, Zapian-Merino SJ and Vazquez-Duhalt R: Bi-enzymatic virus-like bionanoreactors for the transformation of endocrine disruptor compounds. Int J Biol Macromol 146: 415-421, 2020.
3. Pellerin E, Caneparo C, Chabaud S, Bolduc S and Pelletier M: Endocrine-disrupting effects of bisphenols on urological cancers. Environ Res 195: 110485, 2021.

4. Leso V,Fontana L,Marinaccio A,Leopold K, Fanali C, LucchettiD, Sgambato A and Iavicoli I: Sub-chronic palladium nanoparticle effects on the endocrine reproductive system of female Wistar rats: Preliminary data. Toxicol Ind Health 35: 403-409, 2019.

5. Jaffrezic-Renault N, Kou J, Tan D and Guo Z: New trends in the electrochemical detection of endocrine disruptors in complex media. Anal Bioanal Chem 412: 5913-5923, 2020.

6. American Diabetes Association: Classification and Diagnosis of Diabetes: Standards of medical care in diabetes-2020. Diabetes Care 43 (Suppl 1): S14-S31, 2020.

7. Radu L, Carsote M, Gheorghisan-Galateanu AA, Preda SA, Calborean V, Stanescu R, Gheorman V and Albulescu DM: Blood parathyrin and mineral metabolism dinamics: A clinical analyze. Rev Chim 69: 2754-2758, 2018.

8. Zimmet PZ: Diabetes and its drivers: The largest epidemic in human history? Clin Diabetes Endocrinol 3: 1, 2017.

9. Zheng Y, Ley SH and Hu FB: Global aetiology and epidemiology of type 2 diabetes mellitus and its complications. Nat Rev Endocrinol 14: 88-98, 2018.

10. Vann R, Bussuan RM, Rombaldi RL and Arbex AK: Endocrine disruptors and the induction of insulin resistance. Curr Diabetes Rev 17: e102220187107, 2021.

11. Bai Q, Han K, Dong K, Zheng C, Zhang Y, Long Q and Lu T: Potential applications of nanomaterials and technology for diabetic wound healing. Int J Nanomedicine 15: 9717-9743, 2020.

12. Kaur P and Khatik G: An overview of computer-aided drug design tools and recent applications in designing of antidiabetic agents. Curr Drug Targets 22: 1158-1182, 2021

13. Al-Quraishy S, Dkhil MA and Abdel Moneim AE: Anti-hyperglycemic activity of selenium nanoparticles in streptozotocin-induced diabetic rats. Int $\mathbf{J}$ Nanomedicine 10: 6741-6756, 2015

14. Khurana A, Tekula S, Saifi MA, Venkatesh P and Godugu C: Therapeutic applications of selenium nanoparticles. Biomed Pharmacother 111: 802-812, 2019.

15. Deng W, Xie Q, Wang H, Ma Z, Wu B and Zhang X: Selenium nanoparticles as versatile carriers for oral delivery of insulin Insight into synergic antidiabetic effect and mechanism. Nanomedicine 13: 1965-1974, 2017.

16. Kumari S, Kamboj VK, Rajpoot D, Teotia AK, Verma PK and Singh GN: The Unprecedented role of gold nanomaterial in diabetes management. Recent Pat Drug Deliv Formul 13: 219-227, 2019.

17. Mohsen AM: Nanothecnology advanced strategies for the management of diabetes mellitus. Curr Drug Targets 20: 995-1007, 2019.

18. Kumari Y, Singh SK, Kumar R, Kumar B, Kaur G, Gulati M, Tewari D, Gow thamarajan K, Karri VVSNR, Ayinkamiye C, et al: Modified apple polysaccharide capped gold nanoparticles for oral delivery of insulin. Int J Biol Macromol 149: 976-988, 2020.

19. Hou L, Zheng Y, Wang Y, Hu Y, Shi J, Liu Q, Zhang H and Zhang Z: Self-Regulated carboxyphenylboronic acid-modified mesoporous silica nanoparticles with 'touch switch' releasing property for insulin delivery. ACS Appl Mater Interfaces 10: 21927-21938, 2018

20. Zou Z, He D, Cai L, He X, Wang K, Yang X, Li L, Li S and Su X: Alizarin complexone functionalized mesoporous silica nanoparticles: A smart system integrating glucose-responsive double-drugs release and real-time monitoring capabilities. ACS Appl Mater Interfaces 8: 8358-8366, 2016.

21. Iorga RA, Bacalbasa N, Carsote M, Bratu OG, Stanescu AMA, Bungau S, Pantis C and Diaconu CC: Metabolic and cardiovascular benefits of GLP-1 agonists, besides the hypoglycemic effect (Review). Exp Ther Med 20: 2396-2400, 2020.

22. Presas E, Tovar S, Cuñarro J, O'shea JP and O'driscoll CM: Pre-Clinical evaluation of a modified cyclodextrin-based nanoparticle for intestinal delivery of liraglutide. J Pharm Sci 110: 292-300, 2021.

23. Lee YH, Hong YL and Wu TL: Novel silver and nanoparticle-encapsulated growth factor co-loaded chitosan composite hydrogel with sustained antimicrobility and promoted biological properties for diabetic wound healing. Mater Sci Eng C Mater Biol Appl 118: 111385, 2021.

24. Yang L, Zhang L, Hu J, Wang W and Liu X: Promote anti-inflammatory and angiogenesis using a hyaluronic acid-based hydrogel with miRNA-laden nanoparticles for chronic diabetic wound treatment. Int J Biol Macromol 166: 166-178, 2021. 
25. Hu H, Fan X, Guo Q, Wei X, Yang D, Zhang B, Liu J, Wu Q, Oh Y, Feng Y, et al: Silicon dioxide nanoparticles induce insulin resistance through endoplasmic reticulum stress and generation of reactive oxygen species. Part Fibre Toxicol 16: 41, 2019.

26. Rosenfeld CS: Gut dysbiosis in animals due to environmental chemical exposures. Front Cell Infect Microbiol 7: 396, 2017.

27. Farrugia F, Aquilina A, Vassallo J and Pace NP: Bisphenol a and type 2 diabetes mellitus: A review of epidemiologic, functional, and early life factors. Int J Environ Res Public Health 18: 716, 2021.

28. Bakoyiannis I, Kitraki E and Stamatakis A: Endocrine-disrupting chemicals and behaviour: A high risk to take? Best Pract Res Clin Endocrinol Metab 35: 101517, 2021.

29. Khan NG, Correia J, Adiga D, Rai PS, Dsouza HS, Chakrabarty S and Kabekkodu SP: A comprehensive review on the carcinogenic potential of bisphenol A: Clues and evidence. Environ Sci Pollut Res Int 28: 19643-19663, 2021.

30. Vom Saal FS and Vandenberg LN: Update on the health effects of bisphenol A: Overwhelming evidence of harm. Endocrinology 162: bqaa171, 2021.

31. Campbell JE and Newgard CB: Mechanisms controlling pancreatic islet cell function in insulin secretion. Nat Rev Mol Cell Biol 22: 142-158, 2021.

32. Wei Q, Qi L, Lin H, Liu D, Zhu X, Dai Y, Waldron RT, Lugea A, Goodarzi MO, Pandol SJ and Li L: Pathological mechanisms in diabetes of the exocrine pancreas: What's known and what's to know. Front Physiol 11: 570276, 2020.

33. Marraudino M, Bonaldo B, Farinetti A, Panzica G, Ponti G and Gotti S: Metabolism disrupting chemicals and alteration of neuroendocrine circuits controlling food intake and energy metabolism. Front Endocrinol (Lausanne) 9: 766, 2019.

34. Nadal A, QuesadaI,TuduríE,Nogueiras Rand Alonso-MagdalenaP: Endocrine-disrupting chemicals and the regulation of energy balance. Nat Rev Endocrinol 13: 536-546, 2017.

35. Graceli JB, Dettogni RS, Merlo E, Niño O, Da Costa CS, Zanol JF, Ríos Morris EA, Miranda-Alves L and Denicol AC: The impact of endocrine-disrupting chemical exposure in the mammalian hypothalamic-pituitary axis. Mol Cell Endocrinol 518: 110997, 2020.

36. Cocolos AM, Dumitru N, Petrova EN, Cocolos I, Tiglis M, Dragomirescu RFI, Olaru M, Dumitru A and Ghemigian AM: Endocrine disrupting chemicals-the $\mathrm{X}$ factor in different pathologies. Rev Chim 69: 136-139, 2018.

37. Murphy L, Mérida-Ortega Á, Cebrián ME Hernández-Garciadiego L, Gómez-Ruiz H, Gamboa-Loira B and López-Carrillo L: Exposure to bisphenol A and diabetes risk in Mexican women. Environ Sci Pollut Res Int 26: 26332-26338, 2019.

38. Battal D, Cok I, Unlusayin I, Aktas A and Tunctan B: Determination of urinary levels of Bisphenol A in a Turkish population. Environ Monit Assess 186: 8443-8452, 2014.
39. Caban M and Stepnowski P: The quantification of bisphenols and their analogues in wastewaters and surface water by an improved solid-phase extraction gas chromatography/mass spectrometry method. Environ Sci Pollut Res Int 27: 28829-28839, 2020.

40. Kahn LG, Philippat C, Nakayama SF, Slama R and Trasande L: Endocrine-disrupting chemicals: Implications for human health. Lancet Diabetes Endocrinol 8: 703-718, 2020.

41. Lee MR, Kim JH, Choi YH, Bae S, Park C and Hong YC: Association of bisphenol A exposure with overweight in the elderly: A panel study. Environ Sci Pollut Res Int 22: 9370-9377, 2015.

42. Mileva G, Baker SL, Konkle AT and Bielajew C: Bisphenol-A: Epigenetic reprogramming and effects on reproduction and behavior. Int J Environ Res Public Health 11: 7537-7561, 2014.

43. Velmurugan G, Ramprasath T, Gilles M, Swaminathan K and Ramasamy S: gut microbiota, endocrine-disrupting chemicals, and the diabetes epidemic. Trends Endocrinol Metab 28: 612-625, 2017.

44. James-Todd TM, Meeker JD, Huang T, Hauser R, Ferguson KK, Rich-Edwards JW, Mcelrath TF and Seely EW: Pregnancy urinary phthalate metabolite concentrations and gestational diabetes risk factors. Environ Int 96:118-126, 2016.

45. Chevalier N and Fénichel P: Endocrine disruptors: New player in the pathophysiology of type 2 diabetes? Diabetes Metab 41: 107-115, 2015.

46. Shaffer RM, Ferguson KK, Sheppard L, James-Todd T, Butts S, Chandrasekaran S, Swan SH, Barrett ES, Nguyen R, Bush N, et al: Study team. Maternal urinary phthalate metabolites in relation to gestational diabetes and glucose intolerance during pregnancy. Environ Int 123: 588-596, 2019.

47. Zukin H, Eskenazi B, Holland N and Harley KG: Prenatal exposure to phthalates and maternal metabolic outcomes in a high-risk pregnant Latina population. Environ Res 194: 110712, 2021.

48. Chen M, Zhao S, Guo WH, Zhu YP, Pan L, Xie ZW, Sun WI and Jiang JT: Maternal exposure to Di-n-butyl phthalate (DBP) aggravate gestational diabetes mellitus via FoxM1 suppression by pSTAT1 signalling. Ecotoxicol Environ Saf 205: 111154, 2020.

49. Rolfo A, Nuzzo AM, De Amicis R, Moretti L, Bertoli S and Leone A: Fetal-maternal exposure to endocrine disruptors: Correlation with diet intake and pregnancy outcomes. Nutrients 12: 1744, 2020.

50. Feng W, Liu Y, Ding Y, Mao G, Zhao T, Chen K, Qiu X, Xu T, Zhao X, Wu X and Yang L: Typical neurobehavioral methods and transcriptome analysis reveal the neurotoxicity and mechanisms of di(2-ethylhexyl) phthalate on pubertal male ICR mice with type 2 diabetes mellitus. Arch Toxicol 94: 1279-1302; 2020. 